\title{
Paradoxical effects of worrisome thoughts suppression: The influence of depressive
}

\section{$\operatorname{mood}$}

Silva, S., Janeiro, L., Brás, M., Carmo, C., Martins, A.T., \& Jiménez-Ros, A ${ }^{1}$. University of Algarve

\begin{abstract}
Thought suppression increases the persistence of unwanted idiosyncratic worries thoughts when individuals try to suppress them. The failure of suppression may contribute to the development and maintenance of emotional disorders. Depressive people seem particulary prone to engage in unsuccessful mental control strategies such as thought suppression. Worry has been reported to be elevated in depressed individuals and a dysphoric mood may also contribute for the failure of suppression. No studies examine, however, the suppression of worisome thoughts in individuals with depressive symptoms. To investigate the suppression effects of worrisome thoughts, 46 participants were selected according to the cut-off score of a depressive symptomatology scale and they were divided in two groups (subclinical and nonclinical group). All the individuals took part in an experimental paradigm of thought suppression. The results of the mixed factorial analysis of variance revealed an increased frequency of worrisome thoughts during the suppression phase on depending of the depressive symptoms. These findings confirm that depressive mood can reduce the success of suppression.
\end{abstract}

\footnotetext{
${ }^{1}$ Corresponding author: Antónia Jiménez-Ros (PhD, Assistant Professor), Department of Psychology and Educational Sciences, University of Algarve; Campus de Gambelas, 8005-139 Faro, Portugal, email: aros@ualg.pt
} 
Keywords: thought suppression; worrisome thoughts; depressive symptoms, paradoxical effects

\section{Introduction}

Thought suppression is defined as the effort not to think about a particular thought (Wegner, Schneider, Carter, \& White, 1987). This is one of the most used mental control strategies to control unwanted negative thoughts (Iijima \& Tanno, 2012). Paradoxically, however, suppression can increase the intrusiveness of the thoughts intended to be suppressed (Muris, Merckelbach, \& Horselenberg, 1996).

Wegner et al. (1987) found that the suppression of unwanted white bear thoughts led to an intensification of the suppressed thoughts in a post-suppression period (rebound effect). Lavy and van den Hout (1990) also reported an immediate enhancement effect during the thought suppression period when individuals were not allowed to use distraction.

In order to explain the paradoxical effects of thought suppression, Wegner (1994) developed the ironic processes theory of mental control. This theory proposes the existence of two simultaneous mechanisms in thought suppression: (1) an intentional operating process (IOP) that performs a voluntary and conscious search for alternative thoughts (with the purpose of replacing suppression target thoughts and distract unwanted thoughts); and (2) an ironic monitoring process (IMP), automatic and unconscious, that constantly examines possible failures in the IOP. The IOP is responsible for trying to produce efforts to achieve the desired mental state. For this purpose, the operating process needs intensive resources. In case of adjusted cognitive 
resources, thought suppression may not only fail but also prompt the opposite effect, the rebound effect. A dysphoric mood could also hamper the suppression process, resulting in higher ironic effects (Wegner, Erber, \& Zanakos, 1993).

According some authors (e.g., Wenzlaff, Wegner, \& Roper, 1988; Wenzlaff, 2005), depressed individuals are more likely than non-depressed individuals to suffer the ironic effects of suppression due to three main reasons: (a) depressed individuals depend more often on distractors emotionally associated with unwanted thoughts; (b) a depressed mood may consume resources of the operating process; and (c) depressed individuals often experience high levels of subjective stress that can minimize the ability to use distraction effectively.

Wenzlaff et al. (1988) conducted a study with the objective of examine the skills and strategies used by depressed individuals to suppress thoughts. The results of this study were that: (a) depressed individuals use distractors with essentially negative content; (b) they display increased difficulties in thought suppression due to the use of memories related to their unwanted thoughts as a distraction strategy; and (c) they seem to reveal a deficit in the ability to suppress unwanted negative thoughts, experiencing a resurgence of suppressed thoughts as a result of suppression attempts (Wenzlaff et al., 1988).

On the other hand, worry is a transdiagnostic process across depressive and generalized anxiety disorders (Kircanski, Thompson, Sorenson, Sherdell, \& Gotlib, 2015). The suppression of worrisome thoughts also seems to give rise to paradoxical effects (Becker, Rinck, Roth, \& Margraf, 1998) and, at the same time, the perceived failure of thought suppression effort produces an increase of worry associated with the failure of suppression (Beevers, Wenzlaff, Hayes, \& Scott, 1999). 
Iijima and Tanno (2012) conducted a research that aimed to investigate the moderating effect of trait worry on the success of thought suppression related to everyday worries. These authors found that by suppressing their daily worries, individuals with high levels of trait worry reported higher rebound effect levels than individuals with low levels of trait worry.

In conclusion, the tendency towards excessive worry seems to be a transdiagnosis construct common to some anxiety and mood disorders. Thought suppression is a strategy commonly used by individuals to cope with their unpleasant thoughts (including worry); paradoxically, however, this may contribute for the development and maintenance of such disorders. Suppressing thoughts seems to be a difficult task that can be even more difficult if individuals are under dysphoric emotional states. The strategies employed to suppress unpleasant thoughts seem to contribute to the suppression success (i.e., reduction/elimination of the thought to suppress) or failure (i.e., increased frequency of the thought to suppress both during and after suppression).

Given this rationale, we aimed to replicate and extend the paradigm developed by Iijima and Tanno (2012) in depressed and non-depressed participants. With this experimental manipulation, we expect that: (a) paradoxical effects (immediate enhancement and rebound) derive from thought suppression; (b) occurrence of higher ironic processes in the depression group; (c) the nonclinical group present a wider range of resources to deal with the worries; and (d) during the suppression phase, the use of positive, neutral or negative but not related with the most worrisome target thoughts will depend on depressive state.

\section{Material and Methods}




\subsection{Participants}

Forty six Portuguese participants rating in age from 18 to 65 years $(M=43.91, S D$ $=10.81)$ took part of the experiment. Most of the participants were female $(69.60 \%)$, married or living in common law (39.10\%) and of middle socioeconomic status (56.50\%). Concerning to education level, most of the participants hold $12(32.60 \%)$ or $15(23.90 \%)$ years of school.

Participants were recruited from the general population through informal invitation (personal contacts and social networks). The study protocol was approved by the Scientific Committee of the Faculty of Social and Human Sciences of our University.

\subsection{Measures}

2.2.1. Penn State Worry Questionnaire (PSWQ; Meyer, Miller, Metzger, \& Borkovec, 1990; Portuguese version Jiménez-Ros, 2011)

The PSWQ measures the general tendency to excessive worry. Each item consists of five levels of response, in which 1 represents "Not typical at all" and 5 "Very typical". The Portuguese version of the PSWQ-16 items, used in this study, showed an internal consistency of $\alpha=.88$, suggesting a good reliability (Jiménez-Ros, 2011). The internal consistency obtained from the sample in this study was acceptable $(\alpha=.74)$.

2.2.2. Hospital Anxiety and Depression Scale (HADS; Zigmond \& Snaith, 1983; Portuguese version Pais-Ribeiro et al., 2007)

HADS is a 14 items scale with five response levels ranging from 0 to 3 . HADS is divided into two subscales each of them with seven itens: The anxiety subscale (HADSA) and the depression subscale (HADS-D). In the present study, we used the HADS-D. Scores between 0 and 7 were considered "normal", between 8 and 10 "average", 
between 11 and 14 "moderate", and between 15 and 21 "severe". The Portuguese version of the HADS showed a good internal consistency for the depression scale $(\alpha=$ .81) (Pais-Ribeiro et al., 2007). The internal consistency obtained for this study was acceptable $(\alpha=.73)$.

\subsection{Procedure}

The participants signed informed consent, completed a socio-demographic questionnaire, the PSWQ and the HADS-D. Participants were informed that there would be three phases of thought verbalisation but no mention were made that the experience would be related to thought or worry suppression.

We used the HADS-D to screen participants: those who scored above or equal a cutoff score of eight were considered for the subclincal group (SG; $n=24)$ and the participants who scored below of eight for the nonclinical (NG; $n=22)$.

The final sample of 46 was invited to participate in a laboratory experiment. Participants completed the different phases of the study in a quiet room, seated in a comfortable chair and facing the computer screen.

Similarly to Iijima and Tanno's (2012), in our study, participants trained for a three minuts period before the experiment. We asked them to write down a word or phrase that was describing their thoughts when the computer randomly displayed a sound for three times. We programed the Psychopy 2 (Peirce, 2007) to produce the sounds.

Once concluded the training period, the experiment was run in three phases (initial expression, suppression and final expression). During the initial expression phase (IEP), we asked the participants to write their thoughts whenever they heard the sound (exactly as they did during the training period). The computer randomly made a sound 10 times over a period of 10 minutes and the time gap between two consecutive sounds was 40 to 80 seconds. 
After the first phase, the investigator ask the participants to describe their most distressing worrisome thought during the last five days and rate it regarding three aspects: (1) "It's a difficult subject to handle"; (2) "I feel depressed when I think of it"; and (3) "I think regularly on this matter", according to a 7-point scale ranging from 1 (Completely disagree) to 7 (Completely agree).

After that, the investigator asked the participants to rate the similarity between the content of their more most distressing worrisome thought and the content of the spontaneuous thoughts described in the first experimental phase according to a scale with four categories: (1) "This thought is equal to the thought that has worried me during the last days"; (2) "This thought has to do with another matter but is also fundamentally related to worries or negative thoughts"; (3) "This thought has to do with another matter primarily related to neutral or positive themes"; and (4) "I had my mind blank".

Once completed these two tasks, in the suppression phase (SP), we asked the participants to not to think about their most distressing worrisome thought and to write down, again, a word or phrase that reflected what they were thinking when they heard the computer sound. Again, the computer randomly made a sound 10 times over a period of 10 minutes. We requested the classification of their thoughts in accordance with the previous rating scale.

During the final expression phase (FEP), the participants could think about whatever they like. We just required the participants to write down a word or phrase that reflected what they were thinking every time they heard the computer sound, which was again 10 times over a period of 10 minutes. After that, the participants rated their contents thoughts according to the scale used in the previous phases. 
After the experience, we proceeded to an interview to assess the participants' subjective perception about the success, difficulty, and effort spent to suppress their most distressing worrisome thought, as well as the strategies used to suppress the thought. The experience finished with a debriefing about the study objectives.

\subsection{Statistical analysis}

The data were processed with the IBM SPSS software (version 22.0).

The relative frequency of the worrisome target thought was calculated in each phase (i.e., number of thoughts ranked with 1/10). If the supresssion was successful, relative frequency of 1 was low. That means, participant' spontaneous thoughts were not related with their most distressing worrisome thought. If the suppression was not successful, relative frequency was higher, meaning that the participant thought about their most distressing worrisome thought during all the 10 minutes of the suppression phase.

To verify if paradoxical effects of the suppression of idiosyncratic recent daily worries depended on depressive symptoms was performed a mixed ANOVA (3x2). The between groups factor was the depressive symptoms (SG vs. NG) and the within factor was the three experimental phases (IEP, SP, FEP).

For the SP, we also calculated the relative frequency of negative but not related with the target worrisome thoughs and the relative frequency of positive and neutral thoughts (i.e., number of thoughts ranked with $2 / 10$ and 3/10). Low levels of this frequencies means that, during this phase, the participants did not evoque other negative/positive or neutral thoughts to suppress their most distressing worrisome thought. By the opposite, high values (near of 1), means that the participants thought abouts negative/positive and neutral thoughts issues to supress their most worrisome thoughts. To verify if during the SP, the use of positive, neutral or negative but not related with the most worrisome target thoughts will depend on depressive state, we used the relative frequency of 
negative and the relative frequency of positive and neutral thoughts as a factor whithin in a ANOVA (2x2) and the factor between groups was the depressive symptoms.

The partial eta squared $\left(\eta_{\mathrm{p}}{ }^{2}\right)$ was used to estimate the effect size, being values lower than .010 small effect magnitudes, between .058 and .13 average magnitudes, and higher than .13 large magnitudes (Cohen, 1973).

To compare the significance of the differences between two average values for independent samples was used a $t$-test and was considered the value Cohen's $d$ for the effect size estimation. The values of $d$ equal to .2 were considered small effect magnitudes, the values of .5 which were average magnitudes, and the ones higher than .7 were considered large magnitudes (Cohen, 1988).

To compare the significance of the differences between two average values for dependent samples was also used a $t$-test. The magnitude of the difference were calculated using the following formula: $r=\sqrt{\frac{\mathrm{t}^{2}}{\mathrm{t}^{2}+\mathrm{df}}}$ (Field, 2009). The results of the magnitude of the effect were interpreted according to the criteria suggested by the author, where .10 is a weak association, .30 is an average association, and .50 is a strong association.

\section{Results}

\subsection{Manipulation checks}

The subclinical group show a significative $\left(t_{(43)}=2.158, p=.037, d=.66\right.$ ) tendency to pathological worry $(M=53.83, S D=9.45)$ when compared with the nonclinical group $(M=47.95, S D=8.76)$.

We classified the content of the recent daily worries reported by the participants into three themes (academic and labour issues, interpersonal relationships, health and 
welfare). The performed chi-square test indicated that these themes were not associated with the depressive symptoms groups $\left(\chi_{(2)}^{2}=.773, p=.680\right)$.

Both groups differed significantly in the frequency of experience their most distressing worrisome thought $\left(t_{(44)}=2.954, p=.005\right)$, in the difficulty to support them $\left(t_{(44)}=2.893, p=.006, d=.87\right)$, and also in the depressive feelings caused by it $\left(t_{(44)}=2.954, p=.005, d=.89\right)$. The subclinical group presented higher mean values than the nonclinical group in frequency of the most distressing worrisome thought $(M=$ $6.21, S D=.93$ vs. $M=5.27, S D=1.83)$, in the difficulty experienced to support it $(M=$ 5.29, $S D=1.37 v s . M=3.95, S D=1.80)$, and in the depressive feelings caused by these kind of worries $(M=4.83, S D=1.86$ vs. $M=3.09, S D=2.14)$.

The worry trait was not associated with the assessment of most distressing worrisome thought neither in the subclinical group $\left(r_{\text {difficulty }}=.028, n . s ; r_{\text {dysphoria }}=-.196\right.$, $\left.n . s ; r_{\text {frequency }}=.020 ; n . s\right)$ nor in the nonclinical group $\left(r_{\text {difficulty }}=.349, n . s ; r_{\text {dysphoria }}=.216\right.$, $\left.n . s ; r_{\text {frequency }}=.399 ; n . s\right)$.

\subsection{The paradoxical effect of suppression depends from the depressive symptoms}

A mixed ANOVA was performed (3x2) to check whether the paradoxical effects (immediate enhancement and rebound) of the suppression of the most distressing worrisome thought depended on the depressive symptoms (subclinical group vs. nonclinical group). The results suggest that the paradoxical effect of the suppression depends on the depressive symptoms levels $\left(F_{[1.690,74.363]}=3.134, p=.058 ; \eta_{\mathrm{p}}{ }^{2}=\right.$ .066). Although, there were no differences concerning the frequency of the target thought due to depressive symptoms $\left(F_{[1,44]}=1.043 ; p=.313 ; \eta_{\mathrm{p}}^{2}=.023\right)$, the occurrence of the most distressing worrisome thought depends from the experimental phases $\left(F_{[1.690,74.363]}=3.529 ; p=.042 ; \eta_{\mathrm{p}}^{2}=.074\right)$. 
The visual inspection of the Figure 1 suggests that the evolution of the occurrence of the most distressing worrisome thought in the three experimental phases is differentiated on the basis of the depressive symptoms.

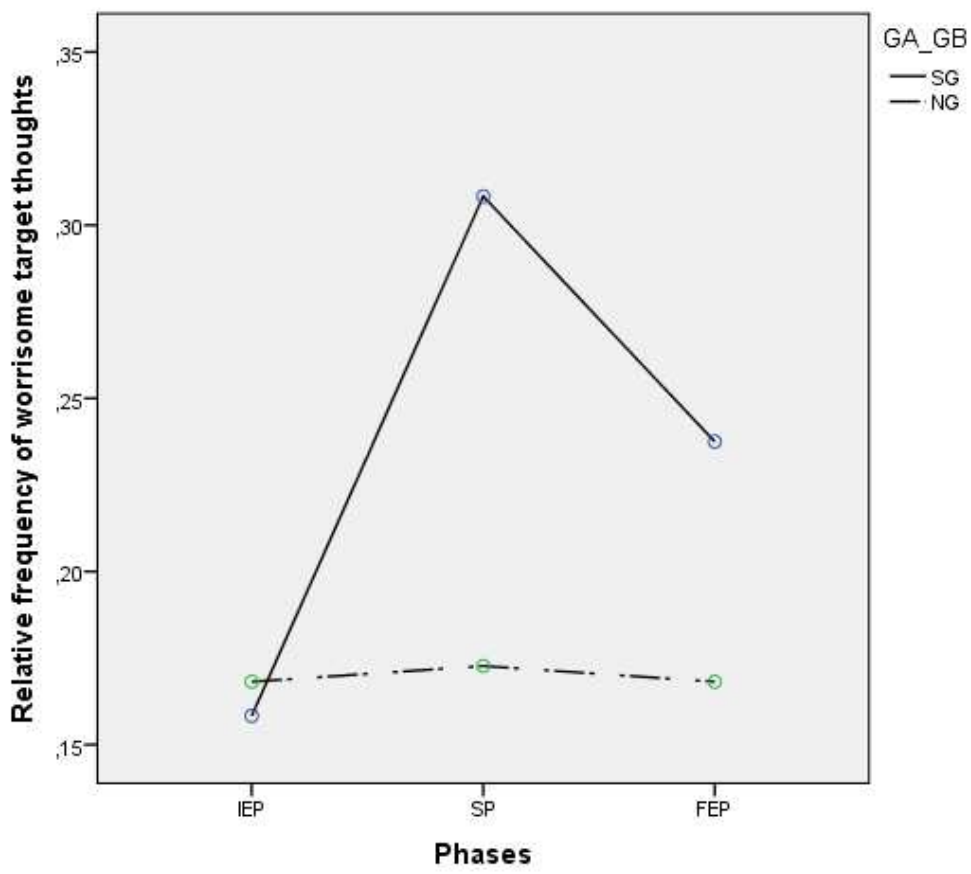

Fig. 1 Evolution of the relative frequency of worrisome thoughts in the three experimental phases, according to the depressive symptoms (SG vs. NG)

In the subclinical group there was a significant increase in the occurrence of the worrisome target thought $\left(t_{(23)}=-3.757, p=.001 ; \alpha \leq .016\right.$ with Bonferroni correction; $r$ $=.62)$ from the IEP $(M=.16, S D=.20)$ to the $\operatorname{SP}(M=.31, S D=.22)$; we further observed a decrease in the frequency of worries from the SP to the FEP $(M=.24, S D$ $=.23)$ that was not significant $\left(t_{(23)}=1.690, p=.105, r=.33\right)$. We also found that the increasing of the frequency of worries from the IEP to the FEP was not significant $\left(t_{(23)}\right.$ $=-1.284, p=.212, r=.25)$. In the nonclinical group the occurrence of the most worrisome thought was very similar in the three experimental phases. There was a nonsignificant increase $\left(t_{(21)}=-.137, p=.892, r=.003\right)$ of the occurrence of the worrisome 
target thought from the IEP $(M=.17, S D=.05)$ to the SP $(M=.173, S D=.06)$; in the FEP it was registered an average value $(M=.17, S D=.06)$ similar to the one found in the IEP.

These results suggest that the depression levels moderated the immediate enhancement suppression effects regarding the worries, namely (a) in subclinical group, the suppression increases the immediate occurrence of the worrisome target thought during the SP; and (b) in nonclinical group, suppression does not affect the occurrence of this thoughts.

3.3. Affective valence (negative vs. positive) of thoughts during suppression phase $(S P)$

In the SP, the affective valence (negative vs. positive) of thoughts which the participants had when they were asked to suppress the most distressing worrisome thought depends on depressive symptoms group $\left(F_{[1,1]}=3.099 ; p=.085 ; \eta_{\mathrm{p}}{ }^{2}=\right.$ .066). The visual inspection of the graph (Figure 2) and the analysis of multiple comparisons ( $\alpha \leq .025$, after Bonferroni correction) suggests that, although, there is more positive thougths in boths groups, in the subclinical group the suppression of an worrisome thought did not lead to the occurrence of significant differences between negative thoughts and positive thoughts $\left(t_{(23)}=-2.003, p=.054, r=.39\right)$; yet, the nonclinical group presented significantly $\left(t_{(21)}=-3.545, p=.002, r=.61\right)$ more thoughts with positive than negative affective valence. 


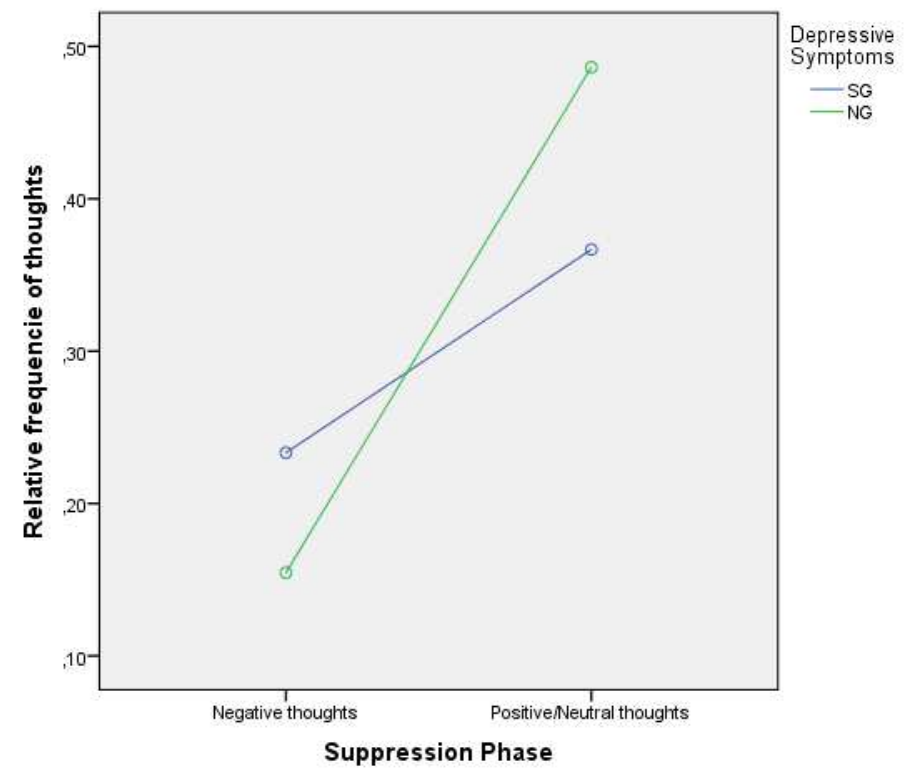

Fig 2 Relative frequency of positive and negative thoughts in the worry suppression phase, according to the depressive symptoms

3.4. Perception of difficulty, effort and success in the suppression phase (SP)

Groups did not differ neither in the perception of difficulty $\left(t_{(44)}=-.680, p\right.$ $=.500, d=-.21)$ nor in the perception of effort expended to suppress the recent daily worries $\left(t_{(44)}=-.200, p=.842, d=-.06\right)$. Nonetheless, the perception of the success was significantly higher for the subclinical group $\left(t_{(44)}=-3.141, p=.003, d=-.95\right)$ lower $(M=3.50, S D=.29)$ than for the nonclinical group $(M=5.10, S D=.42)$.

3.5. Strategies used to cope with the most distressing worrisome thoughts in the suppression phase (SP)

Our data suggest that there was an association between the type of strategies used in the SP to cope with the worrisome thoughts and the groups $\left(\chi_{(2)}^{2}=11.127, p=\right.$ .004). The subclinical group used more cognitive distraction strategies $(91.7 \%)$, while the nonclinical group employed more behavioural distraction strategies $(33.3 \%)$ or did nothing (19.0\%). 


\section{Discussion}

Thought suppression is a strategy commonly used by individuals to cope with their unpleasant thoughts (including worry). The effectiveness of this strategy to reduce unwanted thought frequency is still unclear. To achive this success seems to be more difficult if individuals are under dysphoric emotional states. Our main objective was to test if the success of an idiosincratic worrisome thought suppression may be more difficult under depressive mood. We are also interested to know the most efficient strategies used by the participants to reduce unwanted worrisome thoughts frequency.

In the current study, we used an experiment to suppression considering idiosyncratic worries and to examine the effects of suppressing worrisome thoughts in individuals with and without depressive symptoms.

As expected, we found an association between the presence in trait worry and depression (e.g., McEvoy, Watson, Watkins, \& Nathan, 2013; Starcevic et al., 2007). The subclincal group showed a higher tendency for excessive worry than the nonclinical group.

Although there were no differences in the contents of the most distressing worrisome thoughts experienced by the participants during the days preceding the experience (according on the level of depressive symptoms), the subclinical group rated their primary worry as more frequent, harder to bear and more responsible for dysphoria (when compared to the nonclinical). This result can be explained by metacognitive models that consider the differences between normal and pathological worry as the result of metacognitive beliefs about the same (evaluation of thought, ability to monitor and regulate) rather than the differences in the content of thought (Wells \& Matheus, 1996). It was not associated, however, to the trait of excessive worry, presented in a 
greater extent by the subclinical group. This result provides evidence for Wegner's (1994) perspective, which considers that the suppression affects the occurrence of worrisome thoughts but not the worrisome process itself.

Our results also showed a paradoxical immediate enhancement effect associated to depressive symptoms, and the participants with depressive symptoms presented a higher frequency of thoughts related with their idiosyncratic worry during suppression. This may means that high levels of active negative mood in individuals can deplete the cognitive resources required for suppression (Purdon, 1999). Similar results were obtained by Wegner et al. (1987) and Ashton and Boschen (2011). These authors found that the suppression of unwanted thoughts leads to an immediate enhancement effect on the occurrence of such thought; they further demonstrated that the presence of depressive symptoms has an effect on the failure to suppress worrisome thoughts during the SP. Our findings are also consistent with the results of the study conducted by Conway et al. (1991), who found that, when instructed to suppress thoughts, dysphoric individuals had a higher rate of unwanted thoughts than non-dysphoric individuals. Depressed mood seems to threaten the suppression, allowing unwanted thoughts to return to mind immediately.

Contrary to our predictions, there was no rebound effect; thus, the worrisome thoughts do not seem to return to consciousness once the attempt to suppress them is completed. Our results suggest that, when individuals with dysphoric mood try to suppress a worrisome thought, such attempts become unfruitful only for a short time. Similar results were obtained by Koster, Soetens, Braet, and Raedt (2008), who found that high scores in depression predicted reduced intrusion levels after the suppression. 
The failure to suppress worrisome thoughts is perceived realistically by the participants with depressive symptoms who assessed a minor success to suppress their idiosyncratic worries than participants without depressive symptoms; yet, they did not differ from these latter neither in the effort perceived nor in the difficulty experienced to suppress the worry.

As Murray, Anderson, and Kensinger (2015) concluded in their two experimental studies (comparision memories suppression between young and elderly adults), the failure of the participants' memory suppression appears to be more associated with the failure to select inhibitory strategies than with the incapacity to implement such strategies. In our study, the failure of thought suppression in individuals with depressive symptoms may be related to the type of strategies used to supress the thoughts. In fact, the nonclinical group used a more diverse range of strategies to suppress thoughts (cognitive distraction, behavioural and do nothing), whilst participants with depressive symptoms used mainly the cognitive distraction. Furthermore, the contents of the selected thoughts to implement the distraction during the SP were also different. The participants with depressed mood used more often thoughts with negative content than the participants without depressive symptoms. These results are consistent with those found by Wenzlaff et al. (1988) and may help to explain some results obtained by these authors, who found, in a longitudinal study (Wenzlaff \& Bates, 1998), an association between suppression and high levels of rumination. In a later investigation they further found that the suppression efforts may, ironically, lead to depressive rumination (Wenzlaff \& Luxton, 2003). To confirm these results, future studies are required to manipulate the repertoires of strategies and the content of distractors during the worrisome thought suppression in individuals with or without depression. 
Notwithstanding the relevance of our results, we faced some limitations during the development of this study that did not allow us to draw more general inferences from the data. Some aspects to highlight are the following: (1) the small size of the sample; (2) the heterogeneous group with clinical symptoms; (3) the use of self-report measures may have biased the research results; and (4) finally, and although we did not find an association between the tendency for excessive worry and the individuals' evaluation about their most distressing worrisome thought, this variable may have influenciated the results.

Future research may replicate this study using more homogeneous samples and with diagnosed depression and a superior and representative sample size of the population would allow greater external validity. We also suggested control the role of the variable trait worry in the suppression of worrisome thoughts in individuals with depressive symptoms.

Despite of these limitations, this study is believed to provide valuable insights about a field of increased importance and with clinical and scientific interest. These insights are refered in particularly to the paradoxical effect of immediate enhancement during thought suppression and the importance of negative mood in the suppression of unwanted worrisome thoughts. The participants with depressive symptoms are aware of the failure of thought suppression; yet, they neither assign this task a greater difficulty nor consider making a higher effort than those without depressive symptoms. The lack of diversity strategies and the negative content of the distractors used by the individuals with depressive symptoms may have contributed to the suppression failure. The use of suppression and the type of strategies used to suppress worrisome thoughts can contribute to an increase of rumination and negative mood in these individuals. These results may inform clinicians about the importance of increasing (in therapeutic 
interventions with depressed individuals or with dysphoric mood) the repertoire of coping strategies and changing the contents of distractor thoughts to address the worry.

\section{Compliance with Ethical Standards}

Funding: This study was not funded.

Ethical approval: All procedures performed in studies involving human participants were in accordance with the ethical standards of the institutional and/or national research committee and with the 1964 Helsinki declaration and its later amendments or comparable ethical standards.

Informed consent: Informed consent was obtained from all individual participants included in the study.

\section{References}

Ashton, A., \& Boschen, M. (2011). Thought suppression of multiple personally relevant target thoughts. Asia Pacific Journal of Counselling and Psychotherapy, 2(2), 138-150. doi: 10.1080/21507686.2010.544660.

Becker, E., Rinck, M., Roth, W., \& Margraf, J. (1998). Don't worry and beware of white bears: Thought suppression in anxiety patients. Journal of Anxiety Disorders, 12(1), 39-55. doi:10.1016/j.jbtep.2012.06.003.

Beevers, C., Wenzlaff, R., Hayes, A., \& Scott, W. (1999). Depression and the ironic effects of thought suppression: Therapeutic strategies for improving mental control. Clinical Psychology: Science and Practice, 6(2), 133-148. doi: 10.1093/clipsy.6.2.133. 
Cohen, J. (1973). Eta-squared and partial eta-squared in fixed factor ANOVA designs. Educational and Psychological Measurement, 33(1), 107-112. doi: $10.1177 / 001316447303300111$.

Cohen, J. (1988). Statistical power analisys for the behavioural sciencies (2 ${ }^{\text {nd }}$ Ed.). New York: Academic Press.

Conway, M., Howell, A., \& Giannopoulos, C. (1991). Dysphoria and thought suppression. Cognitive Therapy and Research, 15(2), 153-166.

Field, A. (2009). Discovering Statistics Using SPSS (3 ${ }^{\text {rd }}$ Ed.). London: Sage Publications.

Iijima, Y., \& Tanno, Y. (2012). The rebound effect in the unsuccessful suppression of worrisome thoughts. Personality and Individual Differences, 53(3), 347-350. doi: 10.1016/j.paid.2012.03.023.

Jiménez-Ros, A. (2011). Pensamientos intrusos obsessivos, valoraciones y estratégias de control en individuos no clínicos portugueses (Doctoral thesis). University of Huelva, Spain.

Kircanski, K., Thompson, R. J., Sorenson, J. E., Sherdell, L., \& Gotlib, I. H. (2015). Rumination and worry in daily life: Examining the naturalistic validity of theoretical constructs. Clinical Psychological Science, 3(6) 926-939. doi: $10.1177 / 2167702614566603$.

Koster, E., Soetens, B., Braet, C., \& Raedt, R. (2008). How to control a white bear? Individual differences involved in self-perceived and actual thought-suppression ability. Cogntion and Emotion, 22(6), 1068-1080. doi: $10.1080 / 02699930701616591$.

Lavy, E., \& van den Hout, M. (1990). Thought suppression induces intrusions. Behavioural Psychotherapy, 18(4), 251-258. doi: 10.1017/S0141347300010351. 
McEvoy, P. M., Watson, H., Watkins, E. R., \& Nathan, P. (2013). The relationship between worry, rumination, and comorbidity: Evidence for repetitive negative thinking as a transdiagnostic construct. Journal of Affective Disorders, 151(1), 313-320. doi: 10.1016/j.jad.2013.06.014.

Meyer, T., Miller, M., Metzger, R., \& Borkovec, T. (1990). Development and validation of the Penn State Worry Questionnaire. Behaviour Research and Therapy, 28(6), 487-495.

Muris, P., Merckelbach, H., \& Horselenberg, R. (1996). Individual differences in thought suppression. The white bear suppression inventory: Factor structure, reliability, validity and correlates. Behaviour Research and Therapy, 34 (5), 501513. doi: 10.1016/0005-7967(96)00005-8.

Murray, B. D., Anderson, M. C., \& Kensinger, E. A. (2015). Older adults can suppress unwanted memories when given an appropriate strategy. Psychology and Aging, 30(1), 9-25. doi: 10.1037/a0038611.

Pais-Ribeiro, J., Silva, I., Ferreira, T., Martins, A., Meneses, R., \& Baltar, M. (2007). Validation study of a Portuguese version of the Hospital Anxiety and Depression Scale. Psychology, Health \& Medicine, 12(2), 225-237. doi: $10.1080 / 13548500500524088$.

Peirce, J. W. (2007) PsychoPy - Psychophysics software in Python. J Neurosci Methods, 162(1-2), 8-13.

Pereira, A. (2011). Guia Prático de Utilização do SPSS. Análise de Dados para Ciências Sociais e Psicologia [Practical Guide for Using the SPSS. Data Analysis for Social Sciences and Psychology] ( $7^{\text {th }}$ Ed.). Lisboa: Edições Sílabo.

Purdon, C. (1999). Thought suppression and psychopathology. Behaviour Research and Therapy, 37(11), 1029-1054. doi: 10.1016/S0005-7967(98)00200-9. 
Starcevic, V., Berle, D., Milicevic, D., Hannan, A., Lamplugh, C., \& Eslick, G. D. (2007). Pathological worry, anxiety disorders and the impact of co-occurrence with depressive and other anxiety disorders. Journal of Anxiety Disorders, 21(8), 1016-1027. doi: 10.1016/j.janxdis.2006.10.015.

Wegner, D. (1994). Ironic processes of mental control. Psychological Review, 101(1), 34-52. doi: 10.1037/0033-295X.101.1.34.

Wegner, D., Erber, R., \& Zanakos, S. (1993). Ironic processes in the mental control of mood-related thought. Journal of Personality and Social Psychology, 65(6), 10931104. doi: 10.1037//0022-3514.65.6.1093.

Wegner, D., Schneider, D., Carter, S., \& White, L. (1987). Paradoxical effects of thought suppression. Journal of Personality and Social Psychology, 53(1), 5-13. doi: $10.1037 / / 0022-3514.53 .1 .5$.

Wells, A., \& Matthews, G. (1996). Modelling cognition in emotional disorder: The SREF model. Behaviour Research and Therapy, 34, 881-888.

Wenzlaff, R. M. (2005). Seeking solace but finding despair: The persistence of intrusive thoughts in depression. In D. A. Clark (Ed.), Intrusive Thoughts in Clinical Disorders (pp. 54-85). New York: Guilford Press.

Wenzlaff, R. M., \& Bates, D. E. (1998). Unmasking a cognitive vulnerability to depression: How lapses in mental control reveal depressive thinking. Journal of Personality and Social Psychology, 75(6), 1559-1571. doi: 10.1037/00223514.75.6.1559.

Wenzlaff, R. M., \& Luxton, D. D. (2003). The role of thought suppression in depressive rumination. Cognitive Therapy and Research, 27(3), 293-308. doi: 10.1023/A:1023966400540. 
Wenzlaff, R. M., Wegner, D., \& Roper, D. (1988). Depression and mental control: The resurgence of unwanted negative thoughts. Journal of Personality and Social Psychology, 55(6), 882-892. doi: 10.1037/0022-3514.55.6.882.

Zigmond, A. S., \& Snaith, R. P. (1983). The Hospital Anxiety and Depression Scale. Acta Psychiatrica Scandinavica, 67(6), 361-370. doi: 10.1111/j.16000447.1983.tb09716.x. 